\title{
DOMINATION GAME: EXTREMAL FAMILIES FOR THE 3/5-CONJECTURE FOR FORESTS
}

\author{
Michael A. Henning ${ }^{1}$ \\ Department of Pure and Applied Mathematics \\ University of Johannesburg \\ Auckland Park, 2006, South Africa \\ e-mail: mahenning@uj.ac.za \\ AND \\ Christian LÖWEnstein \\ Institute of Optimization and Operations Research \\ Ulm University \\ Ulm 89081, Germany \\ e-mail: christian.loewenstein@uni-ulm.de
}

\begin{abstract}
In the domination game on a graph $G$, the players Dominator and Staller alternately select vertices of $G$. Each vertex chosen must strictly increase the number of vertices dominated. This process eventually produces a dominating set of $G$; Dominator aims to minimize the size of this set, while Staller aims to maximize it. The size of the dominating set produced under optimal play is the game domination number of $G$, denoted by $\gamma_{g}(G)$. Kinnersley, West and Zamani [SIAM J. Discrete Math. 27 (2013) 2090-2107] posted their 3/5-Conjecture that $\gamma_{g}(G) \leq \frac{3}{5} n$ for every isolate-free forest on $n$ vertices. Brešar, Klavžar, Košmrlj and Rall [Discrete Appl. Math. 161 (2013) 1308-1316] presented a construction that yields an infinite family of trees that attain the conjectured 3/5-bound. In this paper, we provide a much larger, but simpler, construction of extremal trees. We conjecture that if $G$ is an isolate-free forest on $n$ vertices satisfying $\gamma_{g}(G)=\frac{3}{5} n$, then every component of $G$ belongs to our construction.
\end{abstract}

Keywords: domination game, 3/5-conjecture.

2010 Mathematics Subject Classification: 05C57, 91A43, 05 C69.

\footnotetext{
${ }^{1}$ Research supported in part by the South African National Research Foundation and the University of Johannesburg
} 


\section{REFERENCES}

[1] B. Brešar, P. Dorbec, S. Klavžar and G. Košmrlj, Domination game: effect of edgeand vertex-removal, Discrete Math. 330 (2014) 1-10.

doi:10.1016/j.disc.2014.04.015

[2] B. Brešar, S. Klavžar and D.F. Rall, Domination game played on trees and spanning subgraphs, Discrete Math. 313 (2013) 915-923.

doi:10.1016/j.disc.2013.01.014

[3] B. Brešar, S. Klavžar, G. Košmrlj and D.F. Rall, Domination game: extremal families of graphs for the 3/5-conjectures, Discrete Appl. Math. 161 (2013) 1308-1316. doi:10.1016/j.dam.2013.01.025

[4] B. Brešar, S. Klavžar and D. Rall, Domination game and an imagination strategy, SIAM J. Discrete Math. 24 (2010) 979-991. doi:10.1137/100786800

[5] B. Brešar, S. Klavžar and D. Rall, Domination game played on trees and spanning subgraphs, Discrete Math. 313 (2013) 915-923. doi:10.1016/j.disc.2013.01.014

[6] Cs. Bujtás, Domination game on trees without leaves at distance four, in: Proc. 8th Japanese-Hungarian Symposium on Discrete Mathematics and Its Applications, A. Frank, A. Recski, G. Wiener, (Eds), June 4-7, 2013 (Veszprém, Hungary, 73-78).

[7] Cs. Bujtás, S. Klavžar and G. Košmrlj, Domination game critical graphs, Discuss. Math. Graph Theory 35 (2015) 781-796. doi:10.7151/dmgt.1839

[8] P. Dorbec, G. Košmrlj and G. Renault, The domination game played on unions of graphs, Discrete Math. 338 (2015) 71-79. doi:10.1016/j.disc.2014.08.024

[9] M.A. Henning and W.B. Kinnersley, Domination game: A proof of the 3/5conjecture for graphs with minimum degree at least two, SIAM J. Discrete Math. 30 (2016) 20-35. doi:10.1137/140976935

[10] M.A. Henning and A. Yeo, Total Domination in Graphs (Springer Monographs in Mathematics, 2013).

[11] W.B. Kinnersley, D.B. West and R. Zamani, Extremal problems for game domination number, SIAM J. Discrete Math. 27 (2013) 2090-2107. doi: $10.1137 / 120884742$

[12] G. Košmrlj, Realizations of the game domination number, J. Comb. Optim. 28 (2014) 447-461. doi:10.1007/s10878-012-9572-x 